Therapeutics

\section{Erenumab is effective in reducing migraine frequency and improving physical functioning}

10.1136/bmjebm-2018-110937

Check for updates

Paolo Martelletti

Universita degli Studi di Roma La Sapienza Dipartimento di Medicina Clinica, Roma, Italy

Correspondence to: Prof. Paolo Martelletti, Universita degli Studi di Roma La Sapienza; Paolo.Martelletti@uniroma1.it

Commentary on: Goadsby PJ, Reuter U, Hallström YA, et al. A Controlled Trial of Erenumab for Episodic Migraine. $N$ Engl J Med. 2017;377:2123-2132.

\section{Context}

Migraine presents an important social, economic and disability burden in 14\% of affected population. ${ }^{1}$ The age range more compromised is under 50, which represents a burden in personal, social and working functioning. ${ }^{1}$

Migraine treatments are old and with low compliance by the patient for adverse events. Erenumab is a fully human monoclonal antibody that inhibits the calcitonin gene-related peptide receptor (CGRPr) showing promising evidences from a controlled trial in episodic migraine. ${ }^{2}$

\section{Methods}

This randomised, double-blind, multicentre, placebo-controlled trial of erenumab in episodic migraine has been carried out through the monthly subcutaneous administration of either erenumab or placebo for a 6-month period. In total, 955 patients with episodic migraine were divided into three subgroups numerically homogeneous, assigned to erenumab $70 \mathrm{mg}$, erenumab $140 \mathrm{mg}$ and placebo. Primary endpoint was baseline change in the mean number of migraine days per month, from the fourth to the sixth month of therapy.

The secondary outcomes were a reduction in migraine days per month of 50\%, a change in the number of days in which migraine-specific medications were taken and a change in the scores of the test Migraine Physical Function Impact Diary in the specific domains physical impairment and everyday activities.

\section{Findings}

The randomised controlled trial (RCT) reported a reduction in mean migraine days per month during the last three months of treatment of 3.2 days in the erenumab $70 \mathrm{mg}$ group, 3.7 days in the erenumab $140 \mathrm{mg}$ and 1.8 days in the placebo group ( $\mathrm{p}<0.001)$. The same time comparison from baseline to the last three months of treatment has seen a reduction of at least 50\% in mean migraine days per month. The mean number of days in which acute medication was taken was reduced of 1.1 days in the erenumab $70 \mathrm{mg}$ and 1.6 in the erenumab $140 \mathrm{mg}$ groups, while in the placebo group the reduction was of 0.2 days $(p<0.001)$. The evaluation of both dosages of erenumab on patient-reported physical functioning (MPFID) has seen a reduction from baseline scores of 5.5 in erenumab $70 \mathrm{mg}$ and 5.9 in erenumab $140 \mathrm{mg}$ compared with the 3.3 of placebo. MPFID-PI scores resulted in reduction of 4.2 in erenumab $70 \mathrm{mg}$ and 4.8 in erenumab $140 \mathrm{mg}$, compared with the 2.4 of placebo $(\mathrm{p}<0.001)$.

The percent rate of adverse events, although numerically elevated (55.5, 57.3 and 63.0, respectively, for erenumab $70 \mathrm{mg}, 140 \mathrm{mg}$ and placebo), reported no serious safety concerns in all groups, especially with no impact on hepatic function testing.

\section{Commentary}

This trial confirms the efficacy of erenumab in the preventive treatment of migraine through its action of monoclonal antibody towards CGRPr. Clinical improvement and the reduction of the frequency of migraine days are accompanied by a parallel reduction of acute migraine medication, and by a positive effect on the physical functioning of these patients. The safety throughout the 6-month treatment period with both doses of erenumab was very high, with no cardiovascular or hepatic risks. It is clear that RCTs present clean and clear patients, and only a future evaluation of real-world patients undergoing multiple treatments can give further strength to these data. ${ }^{3}$ On the other hand, only the prolonged use of these drugs will show an absolute safety in the clinical daily practice. $^{45}$

\section{Implications for practice}

We are on the edge of a new therapeutic migraine era. We have to understand if this therapy is applicable to any stage of migraine or, like it has been reasonably assumed, it will be directed predominantly to pre-chronic form in order to avoid chronicity that seems to be today the real scourge of this global disease. ${ }^{3}$ Next targets are the comprehension of the interactions between erenumab and acute available drugs such as triptans ${ }^{6}$ and the application of personalised medicine in driving these therapeutic choices. ${ }^{7}$

Competing interests None declared.

Patient consent Not required.

Provenance and peer review Commissioned; internally peer reviewed.

๑ Author(s) (or their employer(s)) 2018. No commercial re-use. See rights and permissions. Published by BMJ.

To cite: Martelletti P. BMJ Evidence-Based Medicine Epub ahead of print: [please include Day Month Year]. doi:10.1136/bmjebm-2018-110937

\section{References}

1. Steiner TJ, Stovner LJ, Vos T, et al. Migraine is first cause of disability in under 50s: will health politicians now take notice? J Headache Pain 2018;19:17.

2. Goadsby PJ, Reuter U, Hallström Y, et al. A Controlled Trial of Erenumab for Episodic Migraine. N Engl J Med 2017;377:2123-32.

3. Martelletti P. The Application of CGRP(r) Monoclonal Antibodies in Migraine Spectrum: Needs and Priorities. BioDrugs 2017;31:483-5.

4. Giamberardino MA, Affaitati G, Costantini R, et al. Calcitonin gene-related peptide receptor as a novel target for the management of people with episodic migraine: current evidence and safety profile of erenumab. J Pain Res 2017;10:2751-60.

5. Deen M, Correnti E, Kamm K, et al. Blocking CGRP in migraine patients - a review of pros and cons. J Headache Pain 2017;18:96.

6. Benemei S, Cortese F, Labastida-Ramírez A, et al. Triptans and CGRP blockade - impact on the cranial vasculature. J Headache Pain 2017;18:103.

7. Pomes LM, Gentile G, Simmaco M, et al. Tailoring Treatment in Polymorbid Migraine Patients through Personalized Medicine. CNS Drugs 2018:559-65. 